\title{
Impact of high-dose rate radiotherapy on B and natural killer (NK) cell polarization in peripheral blood mononuclear cells (PBMCs) via inducing non-small cell lung cancer (NSCLC)-derived exosomes
}

\author{
Qian-Qian Lei ${ }^{1 \#}$, Jiang-Dong Sui ${ }^{1 \#}$, Fu Jin ${ }^{1}$, Huan-Li Luo ${ }^{1}$, Juan-Juan Shan ${ }^{2}$, Li Tang ${ }^{1}$, Ying Wang ${ }^{1}$, \\ Yong-Zhong $\mathrm{Wu}^{1}$ \\ ${ }^{1}$ Radiation Oncology Center, Chongqing University Cancer Hospital, Chongqing, China; ${ }^{2}$ Center for Precision Medicine of Cancer, Chongqing \\ Key Laboratory of Translational Research for Cancer Metastasis and Individualized Treatment, Chongqing University Cancer Hospital, Chongqing, \\ China \\ Contributions: (I) Conception and design: QQ Lei, JD Sui; (II) Administrative support: Y Wang, YZ Wu; (III) Provision of study materials or \\ patients: F Jin, HL Luo; (IV) Collection and assembly of data: QQ Lei, L Tang; (V) Data analysis and interpretation: QQ Lei, JD Sui, JJ Shan; (VI) \\ Manuscript writing: All authors; (VII) Final approval of manuscript: All authors. \\ "These authors contributed equally to this work. \\ Correspondence to: Ying Wang; Yong-Zhong Wu. Chongqing University Cancer Hospital, No. 181 Hanyu Road, Shapingba District, Chongqing \\ 400030, China. Email: yingwang197011@163.com; cqmdwyz@163.com.
}

Background: Non-small cell lung cancer (NSCLC) is the most commonly diagnosed solid tumor. While it has been established that stereotactic body radiotherapy for NSCLC plays an important role in antitumor immune response, the possible effects of the dose rate on this response has not been fully clarified.

Methods: In vitro, A549 cells were irradiated on a Varian TrueBeam ${ }^{\circledR}$ Linear Accelerator with dose and dose rate escalation using the flattening filter-free (FFF) technique, which was followed by coculturing with peripheral blood mononuclear cells (PBMCs). The exosomes from irradiated A549 cells were isolated and then cocultured with PBMCs. Flow cytometry was performed to analyze the proportion of lymph cell clusters in PBMCs.

Results: The proportion of CD3- immune cell clusters in PBMCs was significantly higher in the 10 Gy treatment group than in the nonirradiated group and other lower-dose (2, 6 Gy) treatment groups at the dose rate of 1,000 MU/min. However, no influence was observed on the proportion of CD3+ T cell subsets. Further results showed that both natural killer (NK) and B cell proportions reached peaks in the 14 Gy treatment group when a dose rate of 1,200 MU/min was used. Notably, the peak values of these two cell proportions were reached at a lower radiation dose of 10 Gy when a greater dose rate, ranging from 1,600 to 2,400 MU/min, was used. We further found that a single, high dose of irradiation (10 Gy), as compared with a single, low dose of irradiation (2 Gy), could markedly stimulate the A549-related exosome secretion in a radiation dose rate-dependent manner. The ultrahigh dose rate radiation-derived exosomes contributed to the polarization of B and NK cell subsets in PBMCs.

Conclusions: The optimized radiation regime, which depends on the appropriate radiation dose and dose rate, results in the production of exosomes derived from NSCLC cells and eventually the redistribution of immune cells in PBMCs.

Keywords: Non-small cell lung cancer (NSCLC); ultrahigh dose rate; immune response, stereotactic body radiotherapy; exosome

Submitted Jan 08, 2021. Accepted for publication May 19, 2021.

doi: $10.21037 /$ tcr-21-536

View this article at: https://dx.doi.org/10.21037/tcr-21-536 


\section{Introduction}

Lung cancer, as the most commonly diagnosed malignancy, results in the highest incidence of cancer-related death worldwide (1), with non-small cell lung cancer (NSCLC) accounting for $85 \%$ of all lung cancer cases (2). Although much progress has been made in preclinical and clinical trials for NSCLC therapy, unfortunately, only $15 \%$ achieve 5-year survival after their initial diagnosis.

Distant metastasis is considered to be one of the most critical of the failures in NSCLC therapy. Recent studies have demonstrated that radiotherapy can not only destroy the primary lesion itself but can also eliminate distant metastasis, which is known as the abscopal effect (3); however, the mechanism underlying this effect has not yet been fully explored. It has been revealed that the expression of major histocompatibility complex (MHC) class I molecules involved in antigen presentation are increased after irradiation in a dose-dependent manner (4), implying that the immune system contributes to eliciting radiationrelated abscopal effects (5). In addition, accumulated evidence indicates that stereotactic body radiotherapy is necessary to stimulating a systemic antitumor immune response $(6,7)$. In recent years, ultrahigh-dose rate stereotactic body radiotherapy has become a focal point of research in the field of NSCLC radiotherapy, as this approach has the advantage of being able to deliver a high dose in a short period of time $(8,9)$. However, whether or not ultrahigh-dose rate stereotactic body radiotherapy can augment the antitumor immune response is unknown, while the function of the dose rate in the regulation of the antitumor immune response is also poorly understood.

Many studies have demonstrated that exosomes derived from tumor cells can promote intercellular communication and regulate the immune response in the microenvironment by recruiting immune-related natural killer $(\mathrm{NK})$ cells, $\mathrm{T}$ lymphocytes, and B lymphocytes $(10,11)$. Several recent studies have shown that X-rays not only increase the secretion of exosomes released by tumor cells and the uptake of exosomes by recipient cells, but also lead to changes in the composition of exosomes $(12,13)$. Therefore, the present study was designed to evaluate the effect of ultrahigh-dose rate radiation on the redistribution of immune cells in human peripheral blood mononuclear cells (PBMCs) and to determine whether NSCLC-related exosomes are involved in the radiation-dependent immune response.

We present the following article in accordance with the
MDAR checklist (available at http://dx.doi.org/10.21037/ tcr-21-536).

\section{Methods}

\section{Cell lines, cell culture, and coculture (PBMCs and cell lines)}

An A549 cell line was obtained from the cell bank of the Chinese Academy of Sciences (Shanghai, China) and was cultured in a complete RPMI 1640 medium. Cultures in the log phase of growth were used for all experiments. One day before the irradiation, about $1 \times 10^{6}$ cells per well were seeded in 6-well plates and incubated at $37{ }^{\circ} \mathrm{C}, 5 \% \mathrm{CO}_{2}$ for 24 hours. Transwell inserts with PBMCs (about $10 \times 10^{6}$ cells per insert) were transferred onto A549 cell-containing wells 30 minutes after the irradiation of A549 cells. The cocultured cells were then cultured in the RPMI 1640 medium.

\section{Isolation of PBMCs}

All procedures performed in this study involving human participants were in accordance with the Declaration of Helsinki (as revised in 2013). The study was approved by the Ethics Committee of Chongqing University Cancer Hospital (NO.: CZLS2021042-A) and informed consent was taken from all the patients. Human whole blood was obtained from volunteers. On the same day of A549 cell irradiation, PBMCs were isolated from peripheral blood using Ficoll-Paque PLUS (GE Healthcare Bio-Sciences, USA). To separate PBMCs, $25 \mathrm{~mL}$ of Ficoll were placed in a $50 \mathrm{~mL}$ conical centrifuge tube, and an equal volume of whole blood diluted 1:1 with RPMI1640 was layered onto the Ficoll surface. The $50 \mathrm{~mL}$ tubes were centrifuged at $400 \times \mathrm{g}$ for $30 \mathrm{~min}$ at room temperature. The PBMC interface between the Ficoll and plasma was carefully removed by pipetting and washed twice with phosphatebuffered saline (PBS) by centrifugation at $250 \times \mathrm{g}$ for 10 minutes. After separation, PBMCs were resuspended in RPMI 1640.

\section{Irradiation}

The Varian TrueBeam ${ }^{\circledR}$ Linear Accelerator (Edge) and the flattening filter-free (FFF) technique was used for irradiation (Radiation Oncology Center, Chongqing University Cancer Hospital, Chongqing, China). The instrument was 
set at radiation dose rates of 400, 1,000, 1,200, 1,600, and $2,400 \mathrm{MU} / \mathrm{min}$. The A549 cells were irradiated at doses of 0 , 2, 6, 10, 14, and $18 \mathrm{~Gy}$.

\section{Isolation of exosomes}

The Total Exosome Isolation Reagent (Thermo Fisher Scientific, USA) was used for the isolation of exosomes from irradiated A549 cells. The exosome was isolated following the manufacturer's instructions. The irradiated A549 cells were collected 24 hours after the irradiation and were washed twice with PBS by centrifugation at $300 \times g$ for 5 minutes and 3,000 $\times \mathrm{g}$ for 10 minutes. The isolation solution was added into the supernatant at a 1:2 ratio. The mixed solution was incubated overnight at $4{ }^{\circ} \mathrm{C}$ and then centrifuged at 10,000 $\times \mathrm{g}$ for 1 hour. The exosome was then resuspended in $100 \mu \mathrm{L}$ of PBS and stored at $-20^{\circ} \mathrm{C}$.

\section{Protein determination of exosome}

The protein content of the isolated exosome was determined as recommended by the manufacturer of the Pierce BCA protein assay kit (Pierce Biotechnology, Rockford, IL, USA). Total protein concentrations were determined using a linear standard curve established with bovine serum albumin (BSA). The protein concentrations were calculated as $1 \mu \mathrm{g}$ protein $/ \mu \mathrm{L}$ of plasma.

\section{Coculturing of PBMCs and exosomes}

The exosomes were obtained from the A549 cells and irradiated at a dose of $10 \mathrm{~Gy}$ and a dose rate of 2,400 MU/min after 24 hours of irradiation. PBMCs were also isolated from human whole blood obtained from volunteers. PBMCs and exosomes were cocultured in a $24-$ well plate for 24 hours.

\section{Antibodies and flow cytometry}

PBMCs from the cocultured system were collected and washed twice in PBS, and the cells were then resuspended in $100 \mu \mathrm{L}$ of PBS and incubated for 30 minutes with the following monoclonal antibodies: anti-CD3, anti-CD4, anti-CD8, anti-CD45, anti-CD16, anti-CD56, anti-CD33, anti-CD11c, anti-CD14, anti-CD45RA, and anti-CCR7 antibodies (BD Biosciences, USA) (in the dark at room temperature). Stained samples were analyzed on an LSR II flow cytometer (BD Biosciences). Flow data were quantified using FlowJo software (FlowJo, LLC, Ashland, OR, USA).

\section{Data analysis}

The results are presented as mean \pm standard error (SE). The data were analyzed by statistical software SPSS 21.0 (IBM Corp., Armonk, NY, USA). Student's $t$-test was used for comparison of numeration data, and $\chi^{2}$ test was used for rate comparison of lymphocytes between groups.

\section{Results}

Variation in composition of CD3- immune cell clusters (B and NK cell subsets) in PBMCs induced by A549 cells irradiated at a bigh-dose rate versus a conventional dose rate

A549 cells were cocultured with PBMCs for 2 days upon exposure to increased doses of radiation $(0,2,6$, and $10 \mathrm{~Gy}$ ) using 6-MV $\mathrm{X}$-rays at a dose rate of either 400 or 1,000 MU/min; then, the proportion of CD3immune cell clusters in PBMCs were analyzed by flow cytometry. We found that the proportion of NK cells was significantly higher in the 10 Gy treatment group than in the nonirradiated group $(15.27 \%$ in the 10 Gy group and $11.08 \%$ in the nonirradiated group) and in the other lowerdose treatment groups $(10.76 \%$ in the 2 Gy group and $10.10 \%$ in the 6 Gy group) when A549 cells were irradiated at a dose rate of $1,000 \mathrm{MU} / \mathrm{min}$ (Figure $1 A$ ). However, the variation in the distribution of NK cells was not obviously detected in the increased radiation dose range using a dose rate of $400 \mathrm{MU} / \mathrm{min}$ (Figure 1B). In addition, an influence of the dose rate on the proportion of $\mathrm{B}$ cells was not observed (Figure 1C,D).

\section{Comparison of CD3+T cell subsets in PBMCs induced by $A 549$ cells irradiated at a high-dose rate versus a conventional dose rate}

Next, we compared the effect of A549 cells irradiated with a high-dose rate $(1,000 \mathrm{MU} / \mathrm{min})$ versus a conventional dose rate $(400 \mathrm{MU} / \mathrm{min})$ on the distribution of $\mathrm{CD} 3+\mathrm{T}$ cell subsets. As described above, A549 cells were irradiated at escalating doses (0, 2, 6, and $10 \mathrm{~Gy})$ using 6-MV X-rays at a dose rate of either 400 or $1,000 \mathrm{MU} / \mathrm{min}$ followed by coculture with PBMCs for 2 days. Flow cytometry was performed to analyze the proportion of $\mathrm{CD} 3+\mathrm{T}$ cell clusters in PBMCs. Neither radiation dose escalation nor 
A
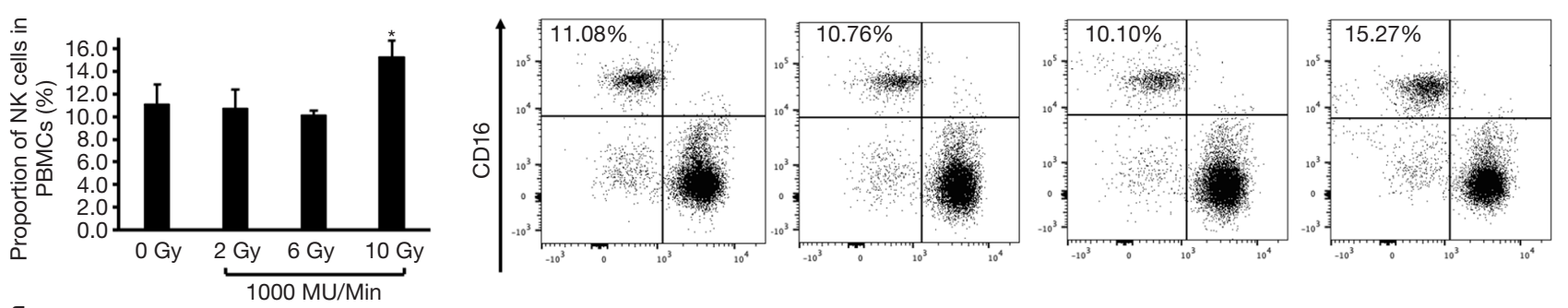

B
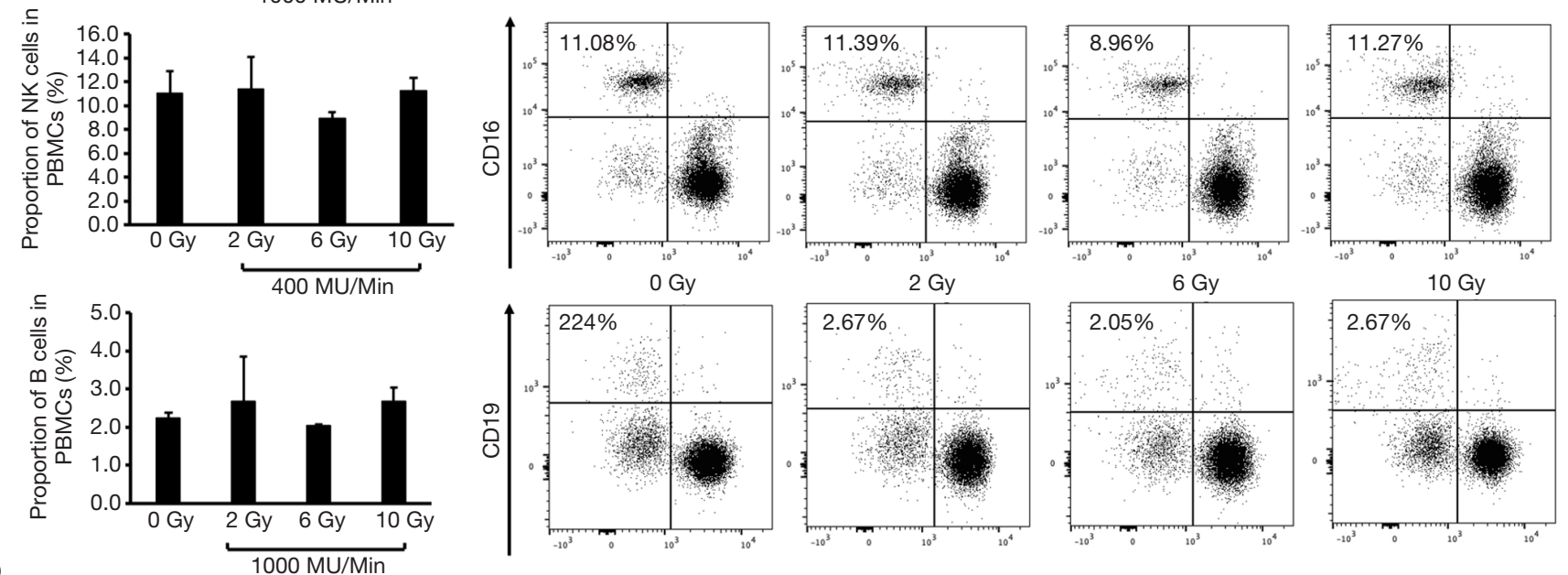

$\mathrm{D}$
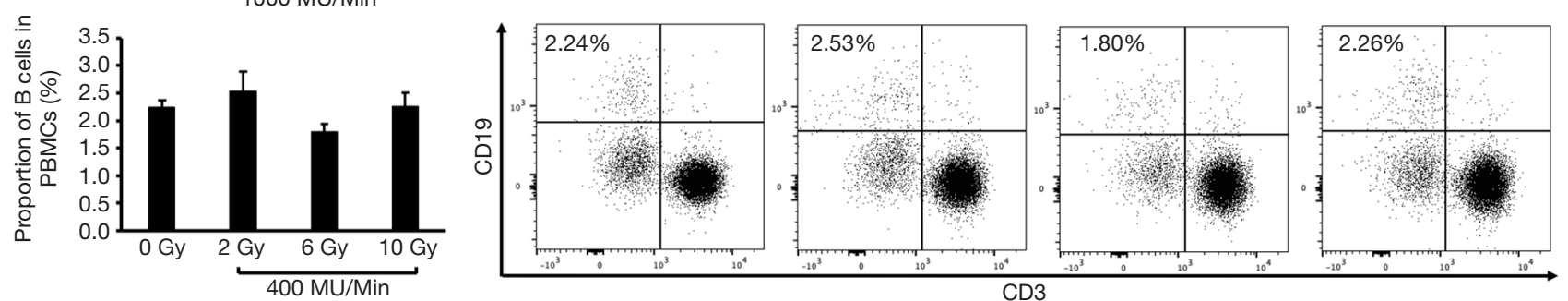

Figure 1 The proportion of changed CD3- immune cell clusters (B and NK cells) in PBMCs after coculture with A549 cells irradiated at various doses with the FFF technique at a high-dose rate and a conventional dose rate. (A) Proportion of NK cells in PBMCs after coculture with A549 cells irradiated at 1,000 MU/min. (B) Proportion of NK cells in PBMCs after cocultures with A549 cells irradiated at $400 \mathrm{MU} / \mathrm{min}$. (C) Proportion of B cells in PBMCs after coculture with A549 cells irradiated at 1,000 MU/min. (D) Proportion of B cells in PBMCs after coculture with A549 cells irradiated at $400 \mathrm{MU} / \mathrm{min} .{ }^{*} \mathrm{P}$ value $<0.05$. FFF, flattening filter-free; PBMC, peripheral blood mononuclear cell; NK, natural killer.

dose rate change was observed to affect the proportion of $\mathrm{CD} 3+\mathrm{T}$ cell subsets, including those of CD4+ T cells, CD8+ $\mathrm{T}$ cells (Figure 2), $\mathrm{T}_{\mathrm{CM}}$ cells, naive cells, $\mathrm{T}_{\mathrm{EM}}$ cells, and $\mathrm{T}_{\text {EMRA }}$ cells (Figure $\mathrm{S} 1$ ).

\section{Influence of high-dose rate radiation on the A549 cell- induced B and NK cell polarization in PBMCs}

Given the effect of a single, high dose of irradiation at a high-dose rate of 1,000 MU/min on A549 cell-induced diversity of CD3- immune cell clusters, we further increased the radiation dose range from 10 to 18 Gy at the dose rate of $1,200,1,600$, and 2,400 MU/min respectively. Flow cytometry was performed to calculate the polarization of $\mathrm{NK}$ and B cell subsets in PBMCs after coculture with irradiated A549 cells. When using the dose rate of 1,200 MU/min, both NK and B cell proportions reached a peak in the 14 Gy treatment group. The proportion of B cells in PBMCs was $4.11 \%, 4.09 \%, 7.70 \%$, and $4.18 \%$ respectively in the 0,10 , 14 , and 18 Gy groups, while the proportion of NK cells in PBMCs was $4.97 \%, 4.18 \%, 7.66 \%$, and $4.46 \%$ respectively in these groups (Figure S2), but the peak values of these 
A
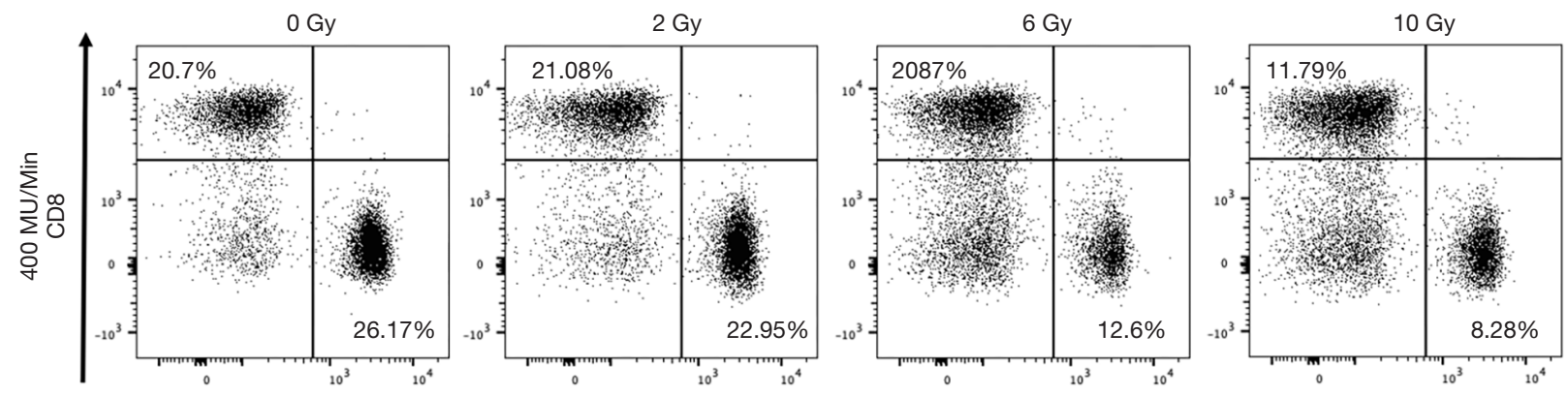

B
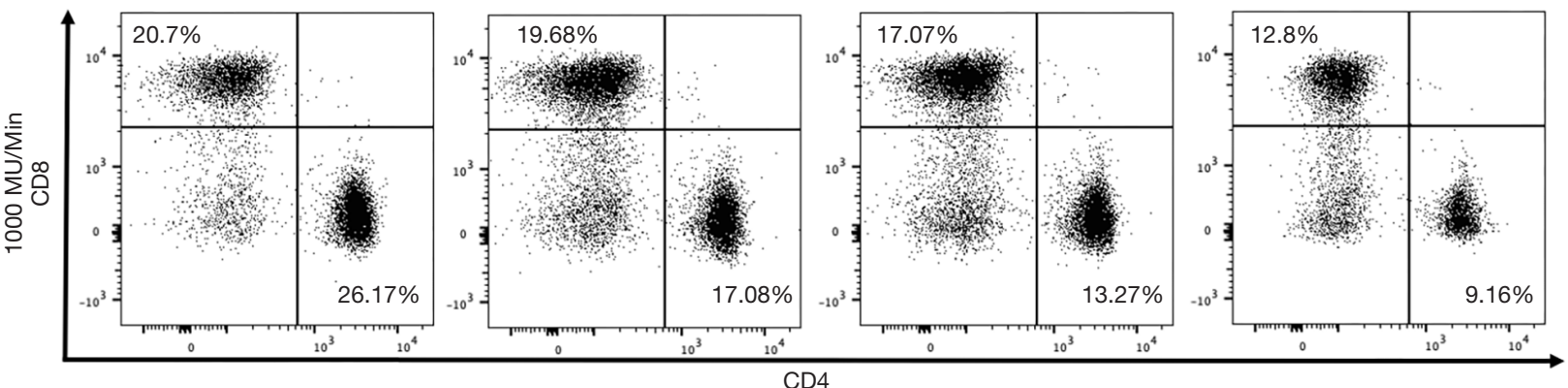

C

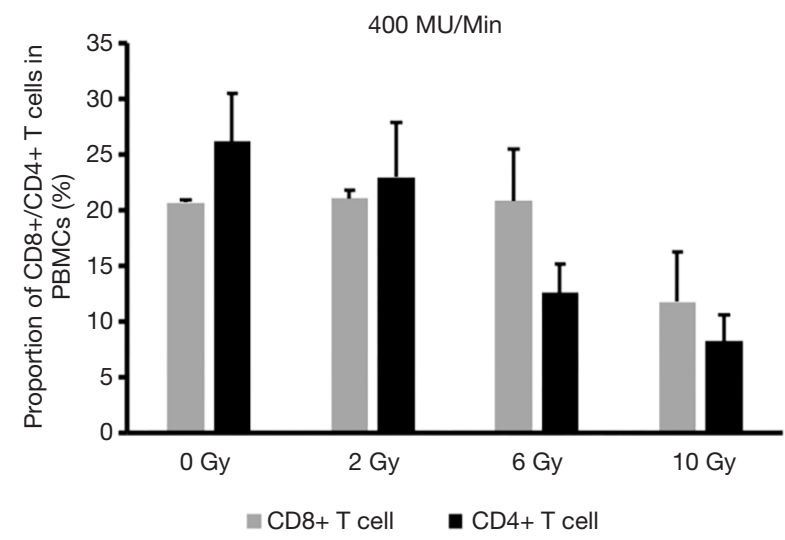

$\mathrm{D}$

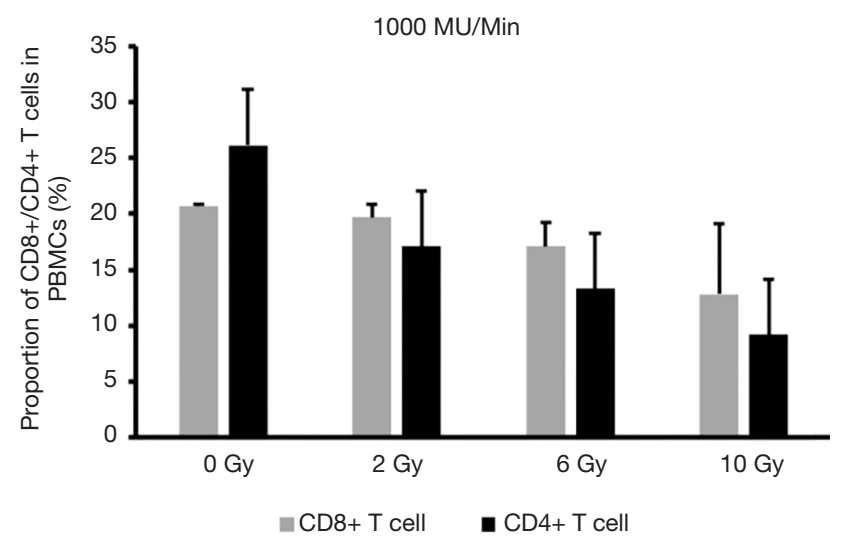

Figure 2 The proportion of $\mathrm{CD} 3+\mathrm{T}$ cell subsets in PBMCs after coculture with A549 cells irradiated at various doses with the FFF technique at a high-dose rate and a conventional dose rate. (A,C) Proportion of CD8+/CD4+ T cells in PBMCs after coculture with A549 cells irradiated at $400 \mathrm{MU} / \mathrm{min}$. (B,D) Proportion of CD8+/CD4+ T cells in PBMCs after coculture with A549 cells irradiated at 1,000 MU/min. PBMC, peripheral blood mononuclear cell; FFF, flattening filter-free; NK, natural killer.

two cell proportions were reached at a lower radiation dose of 10 Gy when a higher dose rate, ranging, from 1,600 to 2,400 MU/min, was used. At a dose rate of 2,400 MU/min (Figure 3), the proportion of B cells in PBMCs was $4.11 \%$, $7.16 \%, 4.15 \%$, and $3.9 \%$ respectively in the $0,10,14$, and 18 Gy groups, while the proportion of NK cells was $4.97 \%$, $7.86 \%, 4.12 \%$, and $4.2 \%$ respectively in these groups (the proportion of B cells was $4.11 \%, 7.69 \%, 3.93 \%$, and $3.60 \%$ in these groups at $1,600 \mathrm{MU} / \mathrm{min}$, while that of $\mathrm{NK}$ cells was $4.97 \%, 7.93 \%, 4.84 \%$, and $3.64 \%$ respectively; Figure S3). Thus, the optimized radiation regime for stimulating A549 cell-induced diversity of CD3-immune cell clusters was determined to be $10 \mathrm{~Gy}$ at a dose rate of 2,400 MU/min.

\section{Influence of high-dose rate radiation on exosomes release from $A 549$ cells}

Growing evidence indicates that ionizing radiation induces an increase in exosome release in a dose-dependent manner, but it is unclear whether the radiation dose rate plays a critical role in promoting the release of exosomes. 
A

B
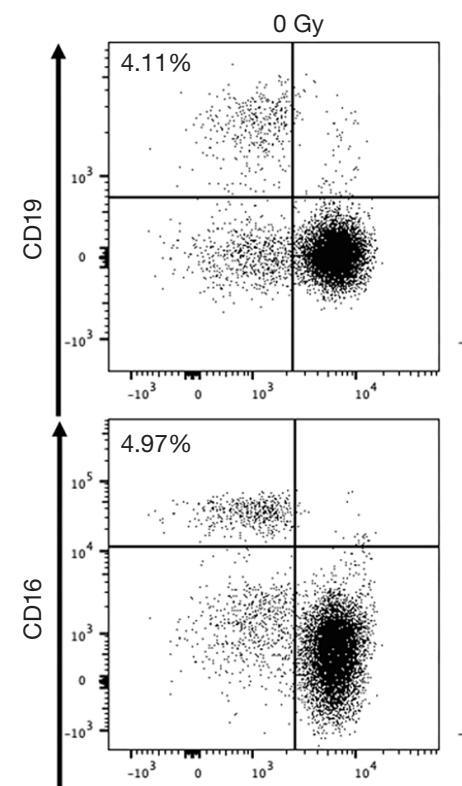
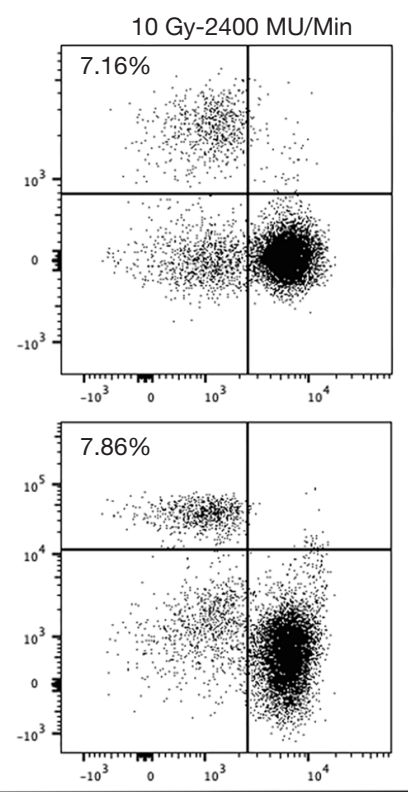
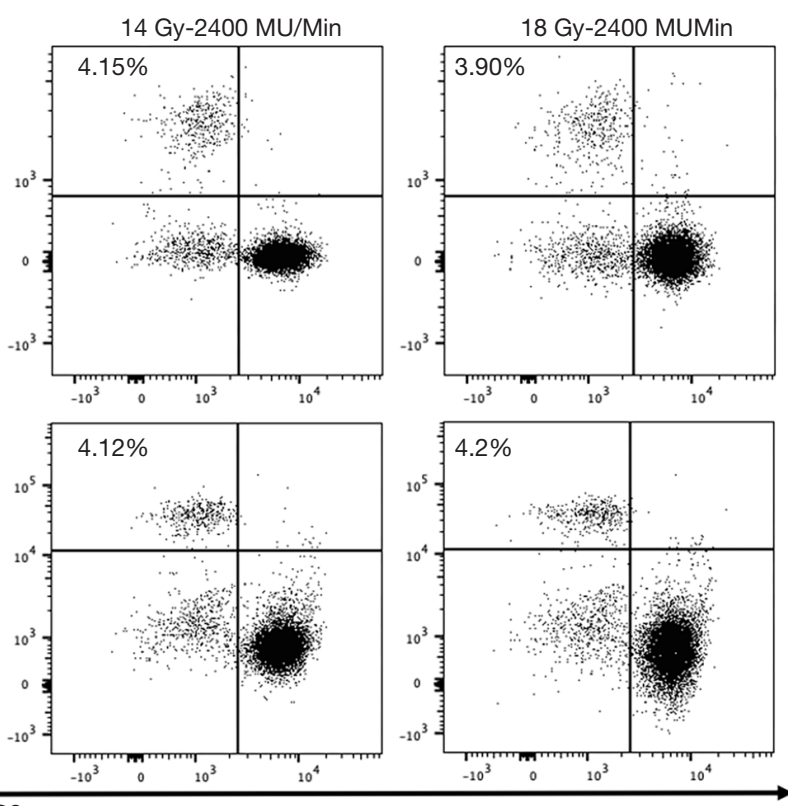

CD3

C
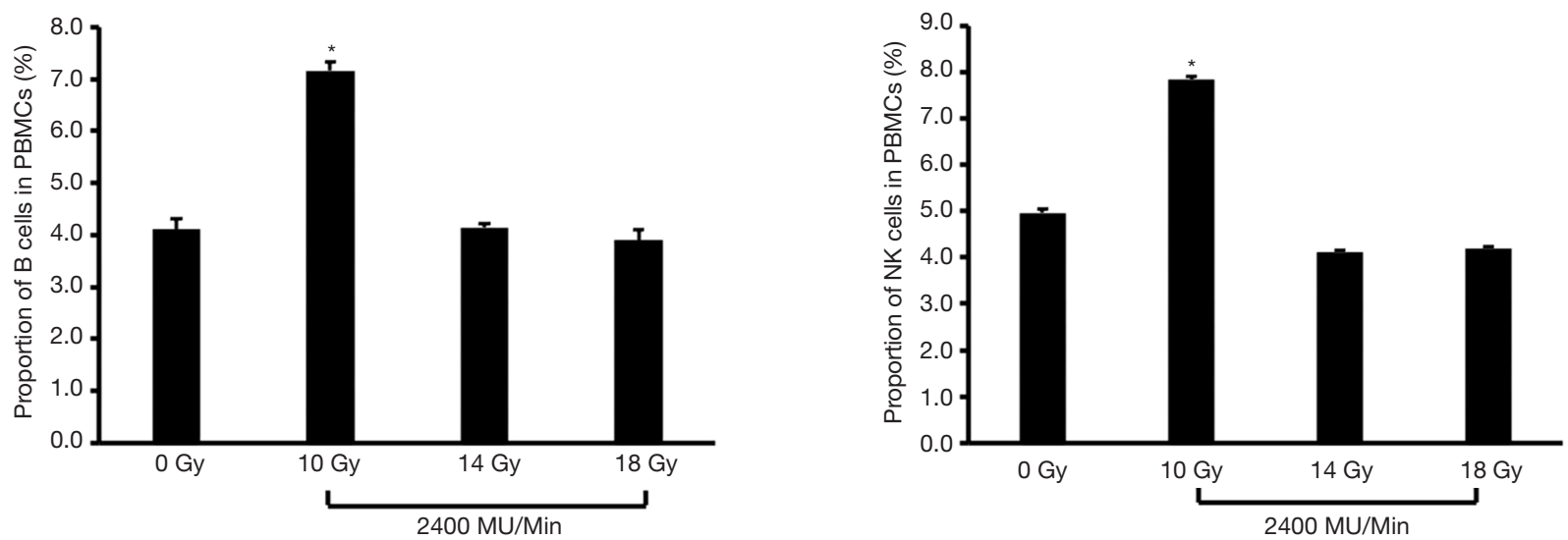

Figure 3 The polarization of B and NK cell subsets in PBMCs after coculture with A549 cells irradiated at various doses with the FFF technique at a high-dose rate $(2,400 \mathrm{MU} / \mathrm{min})$. (A,C) Proportion of B cells in PBMCs after coculture with A549 cells irradiated at 2,400 MU/min. (B,D) Proportion of NK cells in PBMCs after coculture with A549 cells irradiated at 2,400 MU/min. *P value $<0.05$. PBMC, peripheral blood mononuclear cell; NK, natural killer; FFF, flattening filter-free.

Interestingly, a single, high dose of irradiation (10 Gy treatment group) markedly stimulated A549-related exosome secretion in a radiation dose rate-dependent manner (1.01 and 1.12 respectively for the 1,200 and 2,400 MU/min groups) as compared with a single, low dose of irradiation (2 Gy treatment group) (Figure 4). Thus, conventional dose irradiation failed to increase cancerderived exosomes even when the radiation dose rate was elevated (0.92 and 0.83 respectively for the 1,200 and

\section{2,400 MU/min groups) (Figure 4).}

\section{High-dose rate radiation-derived exosomes contributed to the polarization of $B$ and $N K$ cell subsets in PBMCs}

Next, we examined whether the irradiated A549 cell-derived exosomes contributed to the polarization of NK and B cell subsets in PBMCs. Flow cytometry analysis indicated that the proportion of $\mathrm{B}$ and $\mathrm{NK}$ cells were significantly 


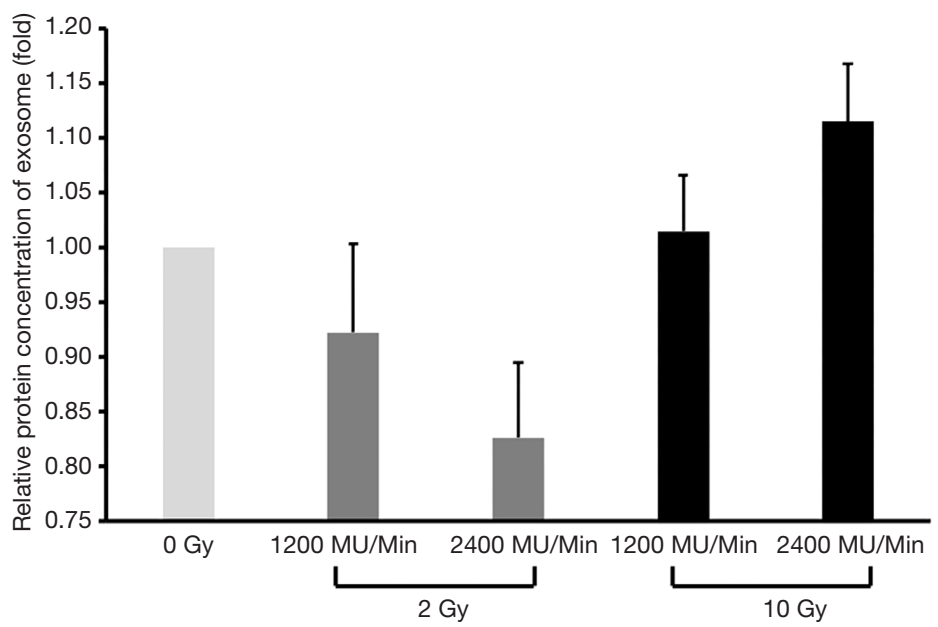

Figure 4 Cellular high-dose rate irradiation increased exosome release.

increased after the PBMCs were directly cocultured with exosomes from the A549 cells irradiated with the optimized regime (10 Gy at a dose rate of 2,400 MU/min). When added with exosomes, the proportion of B cells compared with the group without exosomes (blank group) rose from $5.24 \%$ to $6.87 \%$, while the proportion of NK cells rose from $6.63 \%$ to $8.25 \%$ (Figure 5). These results suggest that the optimized radiation regime not only impacted intercellular communication through exosomes released from exposed donor cells, but also influenced the exosome uptake by the exposed recipient cells.

\section{Discussion}

Exiting studies show that the fractionation and dose rate of irradiation play an important role in regulating immune response in the tumor microenvironment; for example, the recruitment of immune cells into the tumor microenvironment for lesions treated with ultrahigh dose rate stereotactic body radiotherapy is greater than that treated with conventional radiotherapy (CONV-RT) $(14,15)$; however, it is not clear why this is the case. Here, we found that a single, ultrahigh dose of radiation was able to trigger the release of NSCLC-related exosomes and eventually polarize the cell phenotype of PBMCs towards a robust antitumor immune response.

Several experiments have examined ultrahigh dose rate radiotherapy through allowing a higher dose of radiation to be delivered to the lesion at the beginning of clinical treatment $(15,16)$. Another study showed that ultrahigh dose rate stereotactic body radiotherapy could increase the recruitment of immune cells into the tumor microenvironment compared with CONV-RT (14). Our study further demonstrated that the optimal radiation regime to stimulate $\mathrm{A} 549$ cell-induced diversity of CD3- immune cell clusters was $10 \mathrm{~Gy}$ at a dose rate of 2,400 MU/min. On the contrary, one recent study found that a higher dose rate of 16.5 Gy using the FFF technique had no influence on the antitumor immune response (17). Thus, whether or not ultrahigh-dose rate stereotactic body radiotherapy has an effect on the immune system will need to be settled by further research.

An increasing number of studies have shown that exosome shuttling between cells regulates intercellular communication and can alter the phenotype of recipient malignant or stromal cells $(18,19)$. X-ray radiation-induced exosomes can affect angiogenesis through the transport of microRNA (miRNA) to the stromal stem cells, and this biological effect can occur after the addition of the exosome derived from irradiated tumor cells to the stromal stem cells $(20,21)$. Moreover, other recent research has found that $\mathrm{X}$-rays do not only increase the secretion of exosomes released by tumor cells and uptake of exosomes by recipient cells, but also induce changes in component of exosomes $(12,13)$. The present study showed that $\mathrm{X}$-ray radiation with 10 Gy at a dose rate of 2,400 MU/min promoted the release of A549 cell-derived exosomes and further resulted in the polarization of the NK and B cell subsets in PBMCs. An ongoing study of exosome components represents the next step in research in this area for our research team.

In the present work, we found that the optimal radiation regime tended to affect the CD3- $\mathrm{T}$ cell subsets (such 

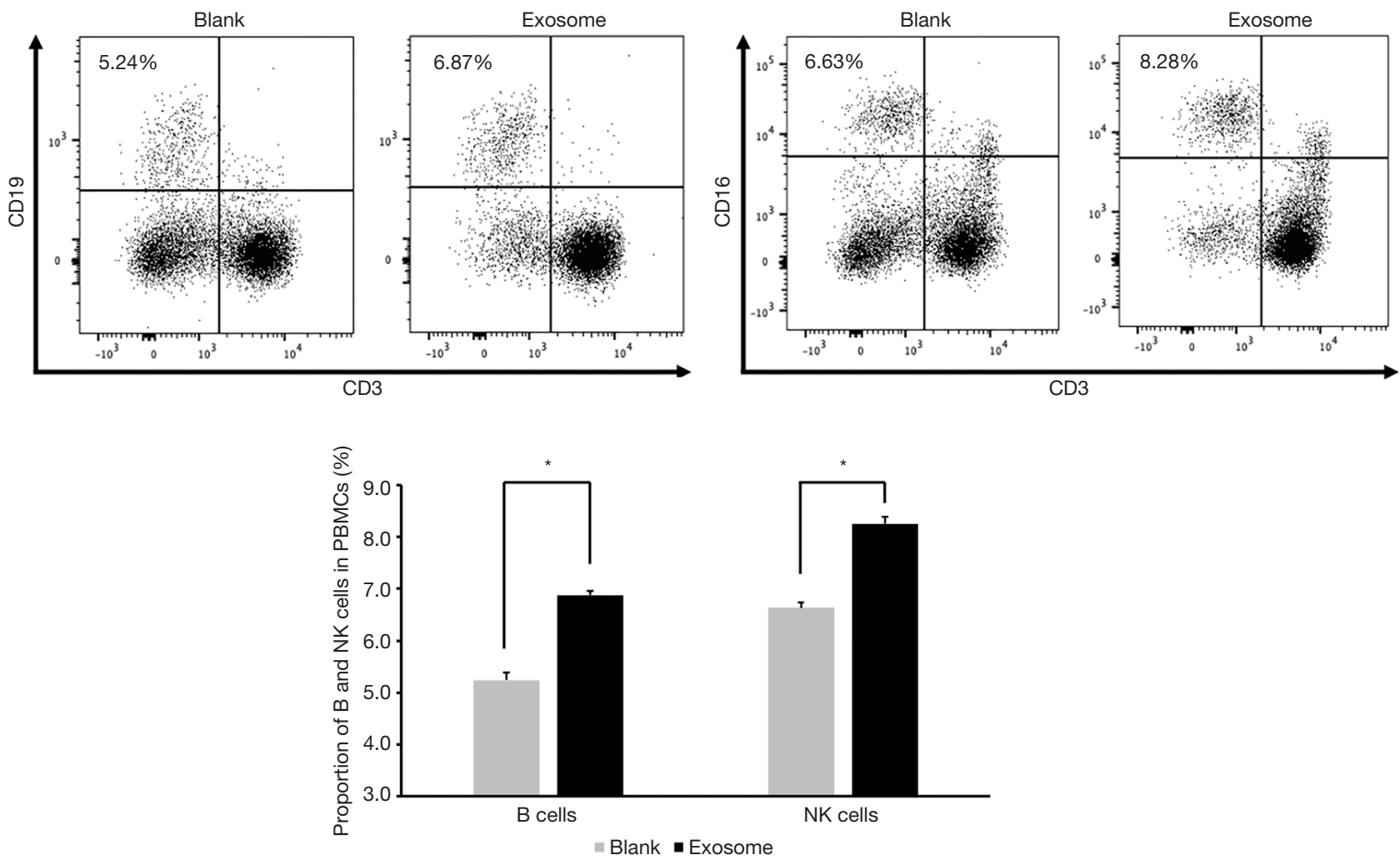

Figure 5 High-dose rate radiation-derived exosomes augmented the polarization of the $\mathrm{B}$ and $\mathrm{NK}$ cell subsets in $\mathrm{PBMCs}$. ${ }^{\mathrm{P}}$ value $<0.05$. NK, natural killer; PBMC, peripheral blood mononuclear cell.

as $\mathrm{NK}$ and $\mathrm{B}$ cells) rather than the $\mathrm{CD} 3+\mathrm{T}$ cell subsets. However, the interpretation of these results may be limited by our methodology, which consisted of an in vitro model. Since the NK cell-mediated killing of tumor cells is independent of $\mathrm{MHC}$ restriction and promotes antigen cross-presentation to CD8+ cytotoxic T cells (22), our in vitro study could only observe the NK cell activation. In addition, other studies have demonstrated that there is a significant association between B cells and CD8+tumorinfiltrating lymphocytes (TILs) and have also found that increased levels of CD8+ and CD4+ TILs colocalizing with high $\mathrm{B}$ cell infiltration are related to long-term survival in NSCLC $(23,24)$; thus, B cells appear to activate earlier than $\mathrm{T}$ cells. We will continue to investigate these issues using in vivo experiments, as they are required to examine the $\mathrm{T}$ cell immune response.

In summary, radiation influences the production of tumor-derived exosomes, which in turn regulate the redistribution of NK and B cells in vitro, with the optimal effect dependent on the appropriate radiation dose and dose rate. Our study sheds light on the effect of ultrahigh-dose rate stereotactic body radiotherapy on the redistribution of immune cells and demonstrates that NSCLC-related exosomes are involved in a radiation-dependent immune response. Our work provides new evidence for exploring the mechanisms by which radiation therapy, especially ultrahigh-dose rate stereotactic body radiotherapy, affects immune response.

\section{Acknowledgments}

The authors would like to thank Yi-Shan Zhang, De-Qing Liu, and Wei Zhou for editing the manuscript.

Funding: This study was supported by the National Natural Science Foundation Project (no. 81802740 to JDS, 82073347 to YZW, 81972857 to YW), the Key Technology Project for Prevention and Control of Major Diseases in Chongqing (Grant No. 2019ZX00), the Chongqing Basic Research and Frontier Exploration Project (Chongqing Natural Science Foundation; no. cstc2018jcyjAX0741), 
the Chongqing Natural Science Foundation Project (cstc2019jcyj-msxmX0648) and the Chongqing Science and Technology Commission Foundation Project (cstc2018jscxmsybX0153).

\section{Footnote}

Reporting Checklist: The authors have completed the MDAR checklist. Available at http://dx.doi.org/10.21037/tcr-21536

Data Sharing Statement: Available at http://dx.doi. org/10.21037/tcr-21-536

Conflicts of Interest: All authors have completed the ICMJE uniform disclosure form (available at http://dx.doi. org/10.21037/tcr-21-536). The authors have no conflicts of interest to declare.

Ethical Statement: The authors are accountable for all aspects of the work in ensuring that questions related to the accuracy or integrity of any part of the work are appropriately investigated and resolved. All procedures performed in this study involving human participants were in accordance with the Declaration of Helsinki (as revised in 2013). The study was approved by the Ethics Committee of Chongqing University Cancer Hospital (NO.: CZLS2021042-A) and informed consent was taken from all the patients.

Open Access Statement: This is an Open Access article distributed in accordance with the Creative Commons Attribution-NonCommercial-NoDerivs 4.0 International License (CC BY-NC-ND 4.0), which permits the noncommercial replication and distribution of the article with the strict proviso that no changes or edits are made and the original work is properly cited (including links to both the formal publication through the relevant DOI and the license). See: https://creativecommons.org/licenses/by-nc-nd/4.0/.

\section{References}

1. Siegel RL, Miller KD, Jemal A. Cancer statistics, 2020. CA Cancer J Clin 2020;70:7-30.

2. Duma N, Santana-Davila R, Molina JR. Non-Small Cell Lung Cancer: Epidemiology, Screening, Diagnosis, and Treatment. Mayo Clin Proc 2019;94:1623-40.

3. Demaria $\mathrm{S}, \mathrm{Ng} \mathrm{B}$, Devitt ML, et al. Ionizing radiation inhibition of distant untreated tumors (abscopal effect) is immune mediated. Int J Radiat Oncol Biol Phys 2004;58:862-70.

4. Reits EA, Hodge JW, Herberts CA, et al. Radiation modulates the peptide repertoire, enhances MHC class I expression, and induces successful antitumor immunotherapy. J Exp Med 2006;203:1259-71.

5. Formenti SC, Demaria S. Systemic effects of local radiotherapy. Lancet Oncol 2009;10:718-26.

6. Lee Y, Auh SL, Wang Y, et al. Therapeutic effects of ablative radiation on local tumor require $\mathrm{CD} 8+\mathrm{T}$ cells: changing strategies for cancer treatment. Blood 2009;114:589-95.

7. Filatenkov A, Baker J, Mueller AM, et al. Ablative Tumor Radiation Can Change the Tumor Immune Cell Microenvironment to Induce Durable Complete Remissions. Clin Cancer Res 2015;21:3727-39.

8. Verma V, Simone CB 2nd. Approaches to stereotactic body radiation therapy for large ( $\geq 5$ centimeter) non-small cell lung cancer. Transl Lung Cancer Res 2019;8:70-7.

9. Thompson M, Rosenzweig KE. The evolving toxicity profile of SBRT for lung cancer. Transl Lung Cancer Res 2019;8:48-57.

10. Zhang HG, Grizzle WE. Exosomes: a novel pathway of local and distant intercellular communication that facilitates the growth and metastasis of neoplastic lesions. Am J Pathol 2014;184:28-41.

11. Whiteside TL. Immune modulation of T-cell and NK (natural killer) cell activities by TEXs (tumour-derived exosomes). Biochem Soc Trans 2013;41:245-51.

12. Arscott WT, Tandle AT, Zhao $S$, et al. Ionizing radiation and glioblastoma exosomes: implications in tumor biology and cell migration. Transl Oncol 2013;6:638-48.

13. Hazawa M, Tomiyama K, Saotome-Nakamura A, et al. Radiation increases the cellular uptake of exosomes through CD29/CD81 complex formation. Biochem Biophys Res Commun 2014;446:1165-71.

14. Rama N, Saha T, Shukla S, et al. Improved tumor control through T-cell infiltration modulated by ultra-high dose rate proton FLASH using a clinical pencil beam scanning proton system. Int J Radiat Oncol Biol Phys 2019;105:S164-5.

15. Loo BW, Schuler E, Lartey FM, et al. (P003) Delivery of Ultra-Rapid Flash Radiation Therapy and Demonstration of Normal Tissue Sparing After Abdominal Irradiation of Mice. Int J Radiat Oncol Biol Phys 2017;98:E16.

16. Favaudon V, Caplier L, Monceau V, et al. Ultrahigh doserate FLASH irradiation increases the differential response 
between normal and tumor tissue in mice. Sci Transl Med 2014;6:245ra93.

17. Laurent PA, Kownacka A, Boidot R, et al. In-vivo and in-vitro impact of high-dose rate radiotherapy using flattening-filter-free beams on the anti-tumor immune response. Clin Transl Radiat Oncol 2020;24:116-22.

18. Bellenghi M, Pontecorvi G, Carè A. On exosome functional role in cancer: miR-494 complex regulation in melanoma cells and corresponding exosomes. Transl Cancer Res 2019;8:725-8.

19. Kalluri R, LeBleu VS. The biology, function, and biomedical applications of exosomes. Science 2020;367:eaau6977.

20. Xu S, Ding N, Pei H, et al. MiR-21 is involved in radiation-induced bystander effects. RNA Biol 2014;11:1161-70.

21. Xu S, Wang J, Ding N, et al. Exosome-mediated

Cite this article as: Lei QQ, Sui JD, Jin F, Luo HL, Shan JJ, Tang L, Wang Y, Wu YZ. Impact of high-dose rate radiotherapy on $\mathrm{B}$ and natural killer (NK) cell polarization in peripheral blood mononuclear cells (PBMCs) via inducing non-small cell lung cancer (NSCLC)-derived exosomes. Transl Cancer Res 2021;10(7):3538-3547. doi: 10.21037/tcr-21-536
microRNA transfer plays a role in radiation-induced bystander effect. RNA Biol 2015;12:1355-63.

22. Krebs P, Barnes MJ, Lampe K, et al. NK-cellmediated killing of target cells triggers robust antigenspecific T-cell-mediated and humoral responses. Blood 2009;113:6593-602.

23. Kinoshita T, Muramatsu R, Fujita T, et al. Prognostic value of tumor-infiltrating lymphocytes differs depending on histological type and smoking habit in completely resected non-small-cell lung cancer. Ann Oncol 2016;27:2117-23.

24. Eerola AK, Soini Y, Paakko P. Tumour infiltrating lymphocytes in relation to tumour angiogenesis, apoptosis and prognosis in patients with large cell lung carcinoma. Lung Cancer 1999;26:73-83.

(English Language Editor: J. Gray) 

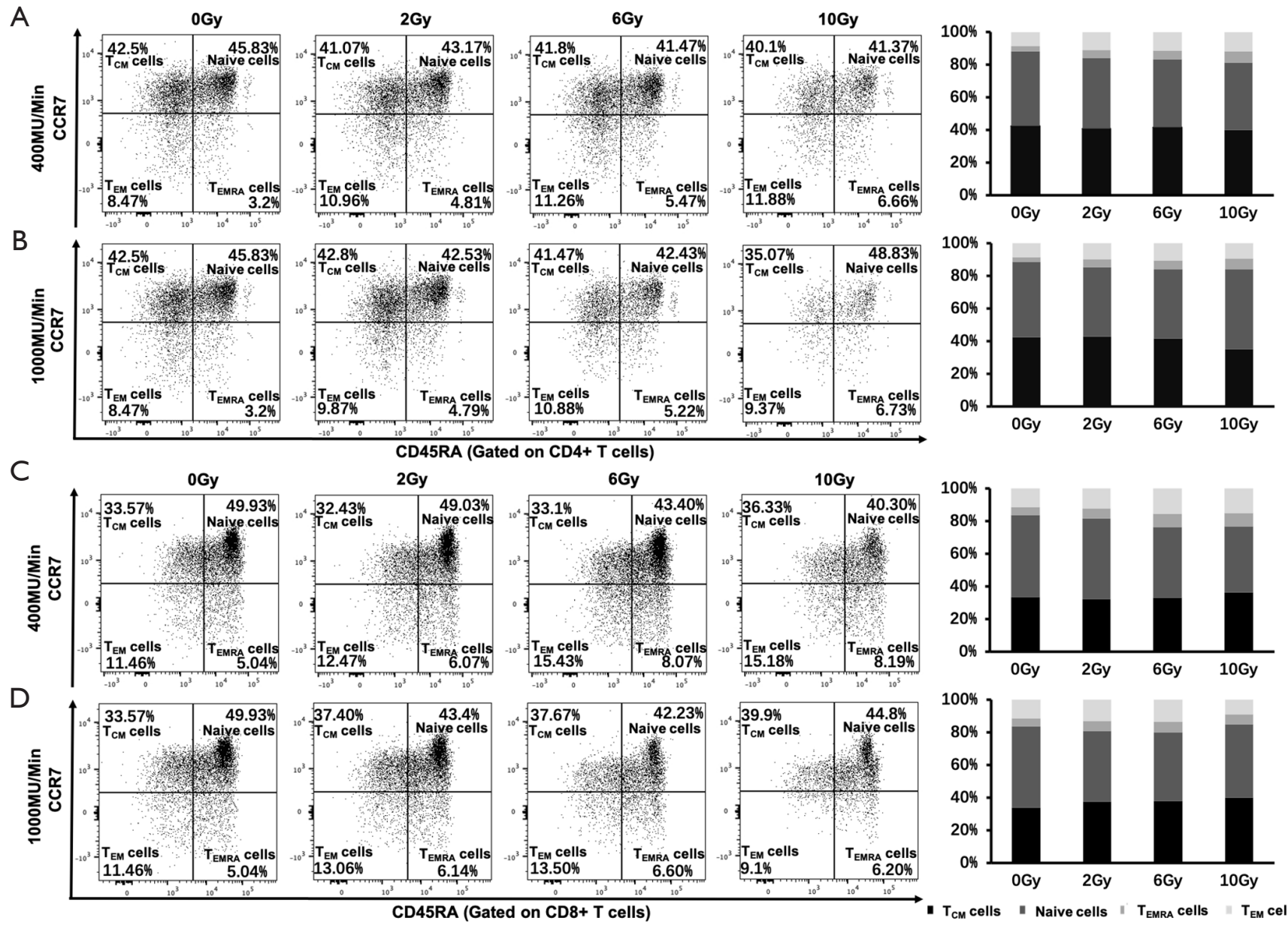

Figure $\mathbf{S 1}$ The proportion of CD4+/CD8+ $\mathrm{T}$ cell subsets ( $\mathrm{T}_{\mathrm{CM}}$ cells, Naive cells, $\mathrm{T}_{\mathrm{EM}}$ cells and $\mathrm{T}_{\mathrm{EMRA}}$ cells) in PBMCs after co-cultured with A549 cells irradiated at various doses with FFF high-dose rate or conventional dose rate. (A) Proportion of CD4+ T cell subsets in PBMCs after co-cultured with A549 cells irradiated at $400 \mathrm{MU} / \mathrm{min}$.(B) Proportion of CD4+ T cell subsets in PBMCs after co-cultured with A549 cells irradiated at 1,000 MU/min. (C) Proportion of CD8+ T cell subsets in PBMCs after co-cultured with A549 cells irradiated at 400 MU/ min. (D) Proportion of CD8+ T cell subsets in PBMCs after co-cultured with A549 cells irradiated at 1,000 MU/min. PBMC, peripheral blood mononuclear cell; FFF, flattening filter-free; NK, natural killer. 
A
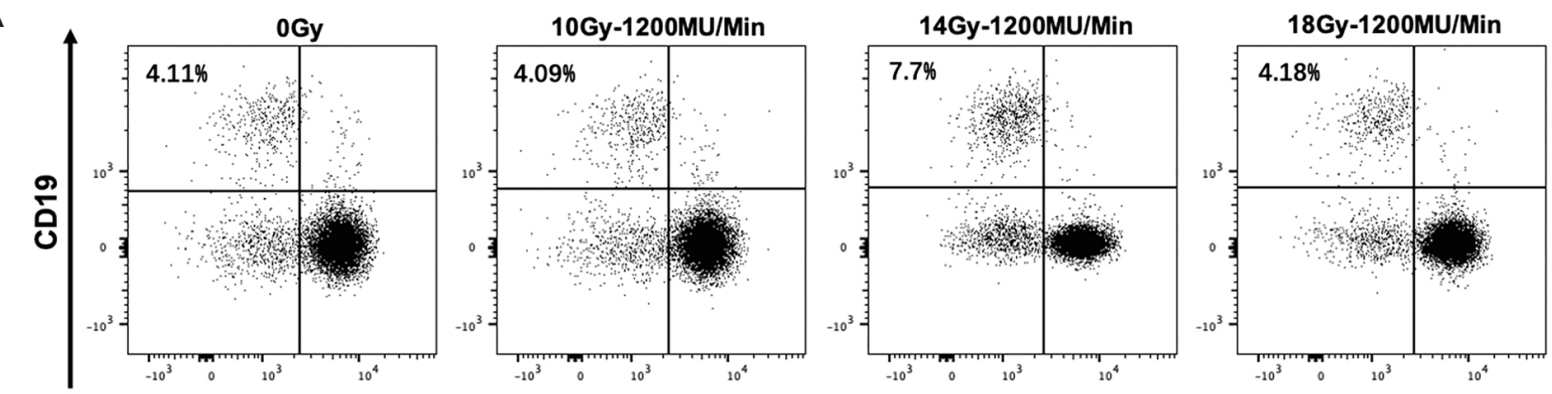

$B$
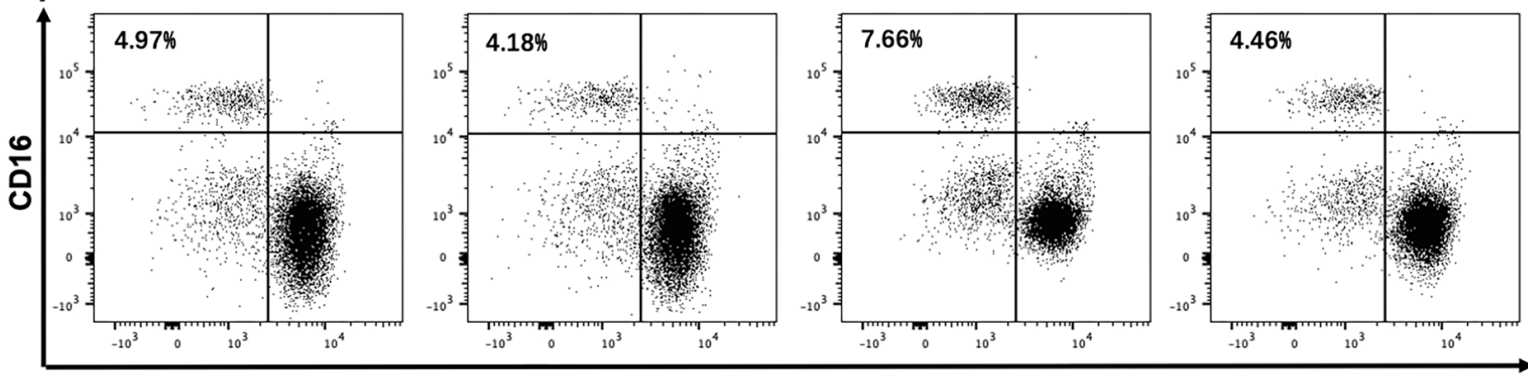

CD3

C
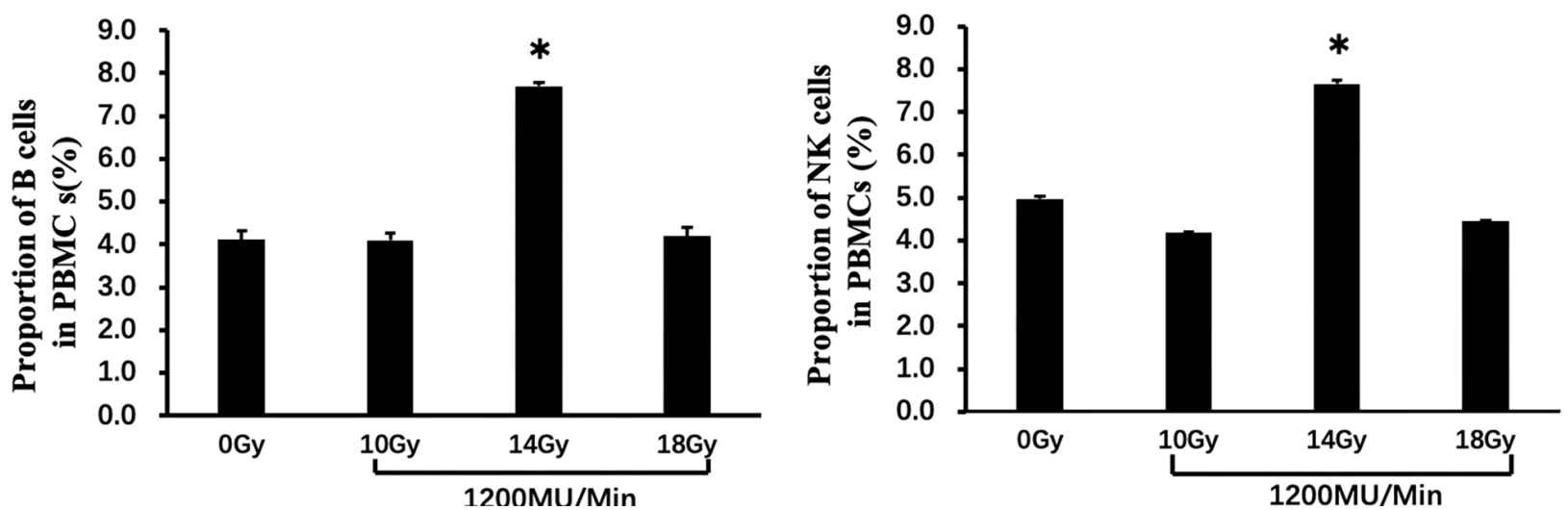

Figure S2 The polarization of B and NK cell subsets in PBMCs after co-culture with A549 cells irradiated at various doses with FFF highdose rate (1,200 MU/min). (A,C) Proportion of B cells in PBMCs after co-cultured with A549 cells irradiated at 1,200 MU/min. (B,D) Proportion of NK cells in PBMCs after co-cultured with A549 cells irradiated at 1,200 MU/min. ${ }^{*} \mathrm{P}$ value $<0.05$. PBMC, peripheral blood mononuclear cell; FFF, flattening filter-free; NK, natural killer. 
A
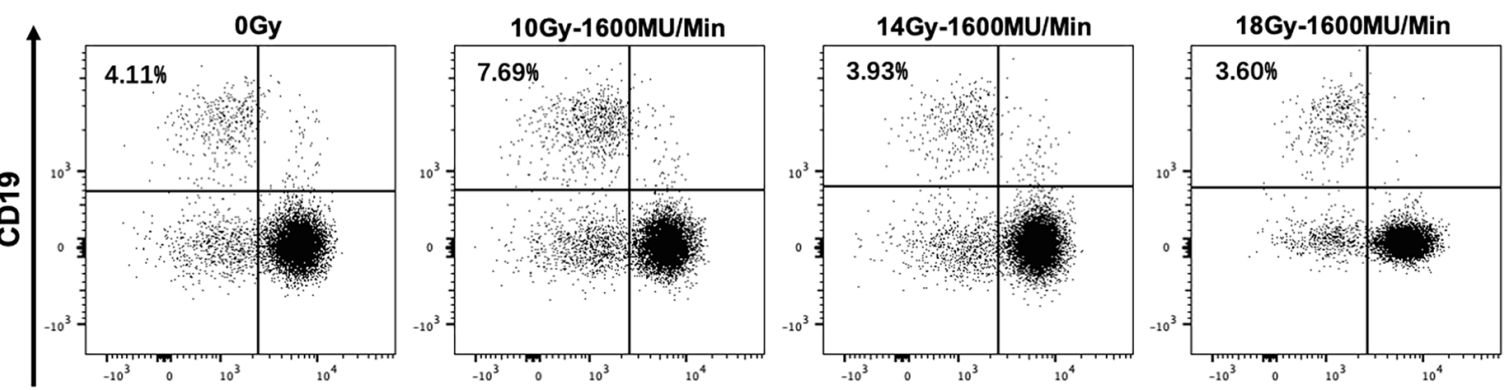

B
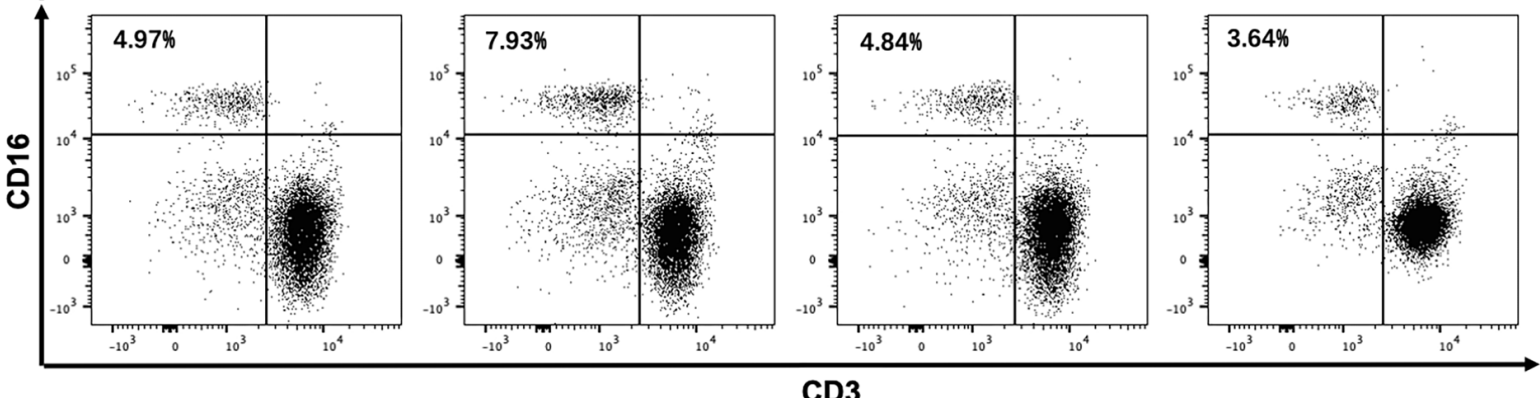

CD3

C

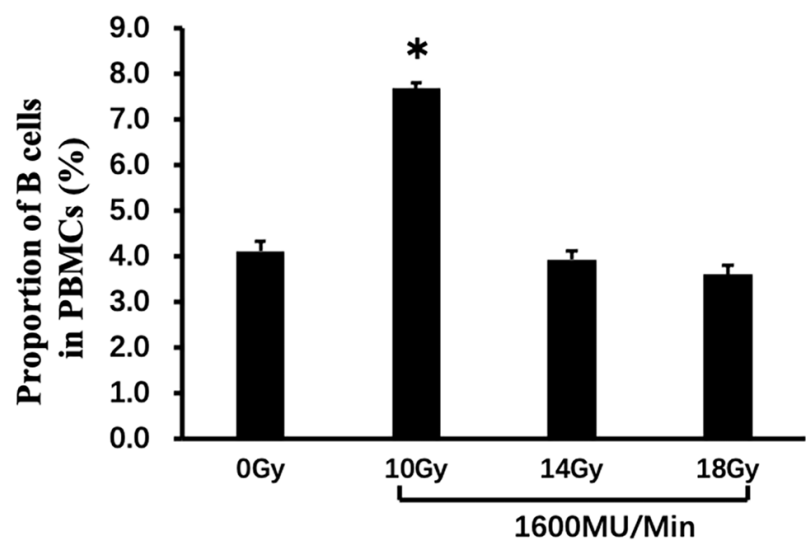

D

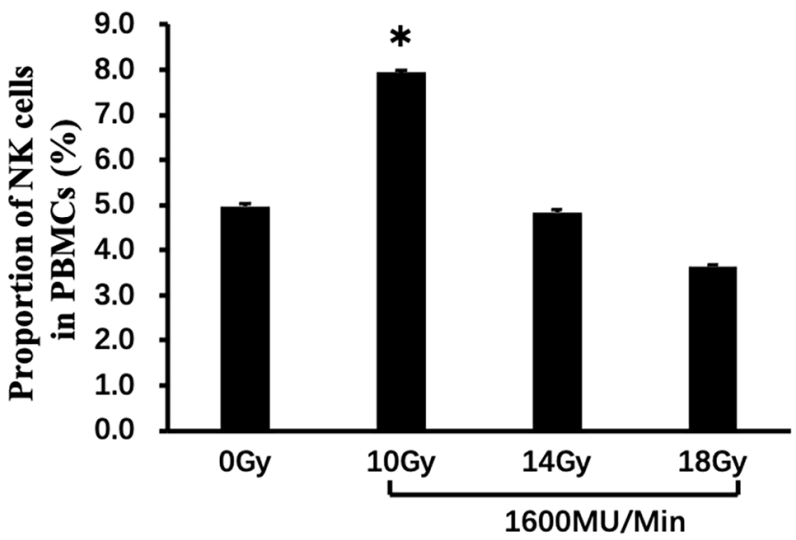

Figure S3 The polarization of B and NK cell subsets in PBMCs after co-culture with A549 cells irradiated at various doses with FFF highdose rate $(1,600 \mathrm{MU} / \mathrm{min})$. (A,C) Proportion of B cells in PBMC after co-cultured with A549 cells irradiated at 1,600 MU/min. (B,D) Proportion of NK cells in PBMCs after co-cultured with A549 cells irradiated at 1,600 MU/min. ${ }^{*} \mathrm{P}$ value $<0.05$. PBMC, peripheral blood mononuclear cell; FFF, flattening filter-free; NK, natural killer. 